\title{
Maintaining dimensions and mechanical properties of ionically crosslinked alginate hydrogel scaffolds in vitro
}

\author{
Catherine K. Kuo, ${ }^{1,2}$ Peter X. Ma ${ }^{1,2,3}$ \\ ${ }^{1}$ Department of Biologic and Materials Sciences, University of Michigan, Ann Arbor, Michigan 48109-1078 \\ ${ }^{2}$ Macromolecular Science and Engineering Center, University of Michigan, Ann Arbor, Michigan 48109-1078 \\ ${ }^{3}$ Department of Biomedical Engineering, University of Michigan, Ann Arbor, Michigan 48109-1078
}

Received 28 August 2006; revised 13 December 2006; accepted 22 January 2007

Published online 23 July 2007 in Wiley InterScience (www.interscience.wiley.com). DOI: 10.1002/jbm.a.31375

\begin{abstract}
Ionically crosslinked alginate hydrogels are attractive scaffolds because of their biocompatibility and mild gelation reaction that allows for gentle cell incorporation. However, the instability of ionically crosslinked hydrogels in an aqueous environment is a challenge that limits their application. This report presents a novel method to control the dimensions and mechanical properties of ionically crosslinked hydrogels via control of the ionic concentration of the medium. Homogeneous calciumalginate gels were incubated in physiological saline baths adjusted to specific calcium ion concentrations. Swelling and shrinking occurred at low and high ionic concentrations of the medium, respectively, while an "optimal" intermediate calcium ion concentration of the medium was found to maintain original size and shape of the hydrogel. This optimal calcium ion concentration was found to be a function of crosslinking density and polymer concentration
\end{abstract}

of the hydrogel and chemical composition of the alginate. The effects of optimal and high calcium ion concentrations of the medium on swelling behavior, calcium content, dry weight, and mechanical properties of the immersed hydrogels were investigated. It was found that the resulting hydrogel composition and mechanical properties depended on not only the calcium concentration of the medium, but also the crosslinking density and polymer concentration of the gel. In an 8-week experiment, controlled dimensions and mechanical properties of alginate gels in an aqueous environment were demonstrated. This new technique significantly enhances the potential of alginate hydrogels for tissue engineering and other biomedical applications. (c) 2007 Wiley Periodicals, Inc. J Biomed Mater Res 84A: 899-907, 2008

Key words: alginate gel; swelling; mechanical property; tissue engineering; scaffold

\section{INTRODUCTION}

Hydrogels are crosslinked networks of hydrophilic polymers that have the capacity to retain large volumes of water. ${ }^{1}$ For certain tissue engineering applications, hydrogels have specific advantages over other scaffold materials, such as their capacity to be mixed with cells in liquid form, and subsequently injected to fill irregularly shaped tissue defects for in situ gelation. ${ }^{2-5}$ Hydrogels are also favorable as carriers for drugs, peptides, and proteins in controlled delivery applications. ${ }^{6}$ Numerous studies have demonstrated the importance of understanding structure-property relationships of hydrogels. ${ }^{7-12}$ With

Correspondence to: P. X. Ma; e-mail: mapx@umich.edu

Contract grant sponsor: Whitaker Foundation; contract grant number: RG-99-0137

Contract grant sponsor: NIH/NIDCR (Biomaterials Training Grant at the University of Michigan School of Dentistry); contract grant number: 2T32DE07057

(C) 2007 Wiley Periodicals, Inc. respect to hydrogels as tissue engineering scaffolds, this knowledge is crucial because material properties affect both scaffold performance and cell function. For example, it has been demonstrated that varying crosslinking density not only alters both compressive modulus and swelling behavior of PEG-based gels, but also affects cell proliferation and matrix molecule synthesis. ${ }^{13-15}$ For drug delivery applications, well-defined structure-property relationships are important, as release kinetics is a function of polymer network swelling. ${ }^{6}$

Alginate hydrogels have shown excellent potential in a variety of biomedical applications, including scaffolds for tissue engineering or carriers for drug delivery systems. ${ }^{16}$ Commonly derived from seaweed, alginate is a linear polysaccharide consisting of $\beta$-D-mannuronic acid (M) and $\alpha$-L-guluronic acid (G) monomers that are arranged in blocks of $G, M$, and random combinations of $\mathrm{M}$ and $\mathrm{G}$ monomers (G-, M-, and MG-blocks). ${ }^{16-18}$ Cooperative crosslinking occurs between calcium ions and G-blocks to result in an "egg-box" structure that imparts gelling 
ability and mechanical strength. ${ }^{19-21}$ The ionic crosslinking reaction is mild and provides a favorable environment for cell immobilization or drug encapsulation. ${ }^{16}$ Traditionally, alginate hydrogels have been crosslinked with $\mathrm{Ca}^{2+}$ to form gel beads (e.g. via dripping alginate solution into a $\mathrm{CaCl}_{2} \mathrm{bath}^{22}$ ) or other shapes (e.g. via mixing alginate solution with $\left.\mathrm{CaSO}_{4}{ }^{23}\right)$. These methods yield gels with structural inhomogeneity because of the uncontrolled rapid crosslinking with $\mathrm{Ca}^{2+} .7,24$ In our previous work, we introduced a novel method to fabricate homogeneous calcium-alginate gels with controllable material properties for tissue engineering scaffolding and other biomedical applications. ${ }^{7}$ We achieved this by controlling the gelation rate of ionically crosslinked alginate gels by balancing the molar ratio of calcium ions derived from a slow gelation reaction $\left(\mathrm{CaCO}_{3}-\right.$ GDL) and that of a fast gelation reaction $\left(\mathrm{CaSO}_{4}\right)$.

However, it is well known that ionically crosslinked hydrogels have a tendency to swell and eventually dissolve in a physiological, aqueous environment. The dimensional stability of ionically crosslinked alginate hydrogels is compromised because of loss of crosslinking calcium ions when in the presence of calcium chelators (e.g. phosphates), monovalent ions (e.g. $\mathrm{K}^{+}, \mathrm{Na}^{+}$, etc.), and noncrosslinking divalent ions (e.g. $\mathrm{Mg}^{2+}$ ) that are present in tissue culture medium and other biological solutions. This phenomenon has been demonstrated by a number of studies characterizing swelling, and more recently, its swelling effects on mechanical properties. A marked decrease in mechanical properties was noted in calcium-alginate gels immersed in physiological baths of $\mathrm{NaCl}$ and $\mathrm{CaCl}_{2} \cdot{ }^{25}$ To overcome this limitation, there have been efforts to stabilize alginate crosslinks. For example, compared to calcium-alginate gels, barium- and copper-crosslinked alginate gels are relatively stable in aqueous solutions. Unfortunately, these cations are often cytotoxic. Alternatively, alginate hydrogels could be covalently crosslinked. However, the reagents involved in the covalent crosslinking reactions are often cytotoxic and provide unfavorable conditions for cell or biofactor incorporation.

Here, we present an alternative to modifying the calcium-alginate gel by describing a novel method to achieve dimensional stability via controlling the calcium ion concentration of a culture environment. We hypothesized that adjusting the $\mathrm{Ca}^{2+}$ concentration in the external environment with respect to the internal $\mathrm{Ca}^{2+}$ concentration would provide control over the swelling behavior and mechanical properties of the gel. To test this hypothesis, we characterized the swelling behavior of these homogenous gels in physiological saline supplemented with specific calcium ion concentrations, and examined the composition and mechanical properties over time. Our objectives were to control the dimensional stability of the gel, to study the effects of the variables of this system on the mechanical properties, and to examine the resultant compositional changes of the gels.

\section{MATERIALS AND METHODS}

\section{Materials}

Sodium alginate prepared from Laminaria hyperborea (LH), Protanal LF200, was from Pronova Biopolymer (Drammen, Norway). High viscosity sodium alginate prepared from Macrocystis pyrifera (MP), calcium carbonate $\left(\mathrm{CaCO}_{3}\right)$, calcium chloride dihydrate $\left(\mathrm{CaCl}_{2} \cdot 2 \mathrm{H}_{2} \mathrm{O}\right)$, calcium sulfate dihydrate $\left(\mathrm{CaSO}_{4} \cdot 2 \mathrm{H}_{2} \mathrm{O}\right)$, and D-glucono- $\delta$-lactone (GDL) were from Sigma (St. Louis, MO).

\section{Alginate gel preparation}

Structurally homogeneous calcium alginate gels were made as previously described. ${ }^{7}$ Sodium alginate was dissolved in deionized water and mixed with calcium carbonate $\left(\mathrm{CaCO}_{3}\right)$ to form a suspension. A fresh aqueous GDL solution was then added to the suspension and vortexed to initiate gelation. $\mathrm{A} \mathrm{CaCO}_{3}$ to GDL molar ratio of 0.5 was maintained to achieve a neutral $\mathrm{pH}$ post-crosslinking. For convenience, a calcium ion to carboxyl molar ratio of 0.18 was designated as $1 \times$. The crosslinking density was adjusted with a multiplication factor to this molar ratio as a relative calcium ion content, such as $2 \times$ (molar ratio: $0.36)$ etc. The gels were cast in Teflon ${ }^{\circledR}$ vials to form circular discs $\sim 8 \mathrm{~mm}$ in thickness and $18 \mathrm{~mm}$ in diameter. The vials were capped, sealed with Parafilm ${ }^{\mathbb{R}}$, and gelled in high humidity at room temperature for $48 \mathrm{~h}$. The indicated alginate gel concentrations in this article were the final weight/volume concentrations.

\section{Swelling experiments}

Swelling behavior of the alginate gels was studied in medium composed of physiological saline adjusted with $\mathrm{CaCl}_{2} \cdot 2 \mathrm{H}_{2} \mathrm{O}$ to varying calcium ion concentrations. Each gel disc was immersed in $15 \mathrm{~mL}$ of medium. The swelling experiments were carried out at room temperature in high humidity by keeping the samples in a bell jar containing a water beaker. The medium was changed every other day. At the scheduled times, the specimens were retrieved and gel surfaces were quickly blotted twice on a Kimwipe ${ }^{\mathbb{R}}$ tissue. The specimens were weighed on an analytical balance accurate to $10^{-4} \mathrm{~g}$. The blotting and weighing process was performed within $1 \mathrm{~min}$ for each specimen. The initial wet weight $\left(W_{\mathrm{o}}\right)$ was obtained after $48 \mathrm{~h}$ gelation. Wet weight $(W)$ during immersion experiment was recorded at designated times. The swelling ratio was defined as $W / W_{0}$. Samples were tested in groups of three. Averages and standard deviations are reported. 


\section{Molecular weight}

The molecular weights of the sodium alginates were calculated from viscosity measurements. The viscosity measurements were performed at $25^{\circ} \mathrm{C}$ with a CannonUbbelohde semimicro viscometer (Cannon Instrument Corporation, State College, PA). Aqueous solutions of 0.03-0.05 g/100 mL sodium alginate with $0.01 \mathrm{M}$ sodium fluoride and $0.09 \mathrm{M}$ sodium chloride were used. Reduced and inherent viscosity, and subsequently intrinsic viscosity, was determined. The molecular weight $(M)$ was calculated using the intrinsic viscosity. ${ }^{7}$

$$
[\eta](100 \mathrm{~mL} / \mathrm{g})=2.0 \times 10^{-5} \mathrm{M}
$$

\section{Mechanical testing}

Uniaxial compression was performed to measure the mechanical properties of the alginate gels with an MTS Synergie 200 mechanical tester (MTS Systems Co., Eden Prairie, MN) at a crosshead speed of $4.8 \mathrm{~mm} / \mathrm{min}$ (initial strain rate of $\left.0.01 \mathrm{~s}^{-1}\right)$. The specimens were coated with silicone oil (Aldrich, Milwaukee, WI) to reduce the effect of friction between the gel and the plates during compression. Eight specimens were tested for each sample. Averages and standard deviations are reported.

\section{Calcium content}

Calcium content of the gels was determined with a colorimetric diagnostic kit (catalog 587-A) from Sigma (St. Louis, MO). Gels were quickly blotted on a Kimwipe ${ }^{\circledR}$ tissue and then transferred to clean, dry crucibles for burning in a Jelrus Cera-Mat III vacuum furnace. To avoid bursting gels with extremely high water content, specimens were first brought to higher temperature by ramping to $427^{\circ} \mathrm{C}$ (rate: $1^{\circ} \mathrm{C} / \mathrm{min}$ ) and then held for $1 \mathrm{~h}$. The temperature was then increased to $732^{\circ} \mathrm{C}$ (rate: $1^{\circ} \mathrm{C} / \mathrm{min}$ ) and held for $20 \mathrm{~min}$ before ramping back down and allowing the crucible with sample to cool to room temperature. The remaining ashes were then dissolved in $1 \mathrm{M} \mathrm{HCl}$. The calcium concentration of the $\mathrm{HCl}$ solution was then determined as per the manufacturer's protocol with absorbance measurements taken at $575 \mathrm{~nm}$ on a Shimadzu UV-265 UV-Vis spectrophotometer (Columbia, MD). Three samples were tested for each group. Averages and standard deviations are reported.

\section{Polymer content}

To estimate polymer content, dry weight was determined by weighing the gel after drying at $37^{\circ} \mathrm{C}$ for $48 \mathrm{~h}$ (constant value reached). The calcium content (determined above) was then subtracted from the total dry weight to obtain the alginate dry weight $\left(W_{\mathrm{d}}\right)$. Dry to original-wet-weight ratios were calculated as the ratio of $W_{\mathrm{d}} / W_{\mathrm{o}}$. The weighing procedure of wet gels was as described for the swelling experiments. Three samples were tested for each group. Averages and standard deviations are reported.

\section{Statistical analysis}

A two-tail Student $t$-test (assuming unequal variances) was performed to determine the statistical significance $(p<0.05)$.

\section{RESULTS}

\section{Swelling as a function of calcium concentration in the medium}

LH alginate gels (1.5\%) with $2 \times$ initial crosslinking density $\left(\mathrm{CaCO}_{3}\right)$ were immersed in media composed of physiological saline with varying calcium ion concentrations of 1.0-5.0 $\mathrm{mM} \mathrm{Ca}{ }^{2+}$ for 2 weeks. The swelling profiles (wet weight ratio $\left(W / W_{\mathrm{o}}\right)$ vs. time) were obtained by monitoring weight change during immersion (Fig. 1). A low $\mathrm{Ca}^{2+}$ concentration (e.g., $1.0 \mathrm{mM}$ ) of the medium resulted in swelling of the gels, while a high $\mathrm{Ca}^{2+}$ concentration of the medium resulted in shrinkage of the gels. After immersion in medium for 2 weeks, swelling ratios reached stable values with little additional change.

\section{Swelling as a function of gel crosslinking density}

To examine the effect of initial crosslinking density of a gel on swelling behavior, swelling profiles similar to Figure 1 (data not shown) were obtained for $1.5 \% \mathrm{LH}$ alginate gels with varying crosslinking densities $\left(1,1.5,2,3\right.$, and $\left.4 \times \mathrm{CaCO}_{3}\right)$ immersed in medium with varying $\mathrm{Ca}^{2+}$ concentrations for 2 weeks. It was consistently found that for all gels with varying initial crosslinking densities, swelling

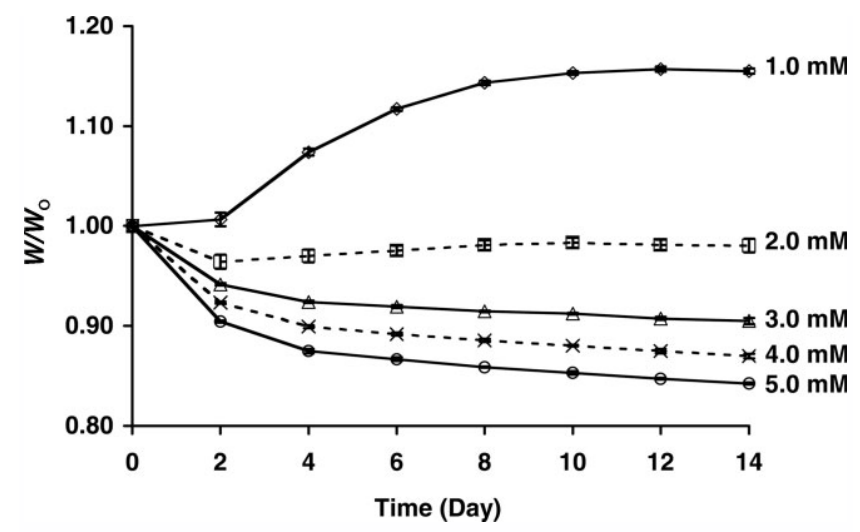

Figure 1. Typical swelling behavior of alginate gels immersed in medium of varying $\mathrm{Ca}^{2+}$ concentrations (1.0$\left.5.0 \mathrm{mM} \mathrm{CaCl} \cdot 2 \mathrm{H}_{2} \mathrm{O}\right)$. The gels swelled $\left(W / W_{\mathrm{o}}>1\right)$ at low $\mathrm{Ca}^{2+}$ concentrations and shrank $\left(W / W_{\mathrm{o}}<1\right)$ at high $\mathrm{Ca}^{2+}$ concentrations. The data presented here characterizes $1.5 \%$ $\mathrm{LH}$ alginate gels with $2 \times \mathrm{CaCO}_{3}$. 
occurred at lower $\mathrm{Ca}^{2+}$ concentrations (e.g. $1.0 \mathrm{mM}$ ), while shrinking occurred at higher (e.g. $5.0 \mathrm{mM}$ ) $\mathrm{Ca}^{2+}$ concentrations, with all reaching stable values by 2 weeks [Fig. 2(a)]. "Optimal" $\mathrm{Ca}^{2+}$ concentrations of the medium at which each gel composition would maintain original dimensions $\left(W / W_{\mathrm{o}}=1\right)$ were determined by fitting the data at 2 weeks with regression curves based on a power relation (Table I). The dependency of optimal $\mathrm{Ca}^{2+}$ concentration on crosslinking density $\left(\mathrm{CaCO}_{3}\right.$ content of the original gels) was found to exhibit a biphasic nature, where both low and high initial crosslinking densities required high optimal $\mathrm{Ca}^{2+}$ concentrations, whereas intermediate crosslinking densities required low optimal $\mathrm{Ca}^{2+}$ concentrations.

\section{Swelling as a function of polymer concentration}

To study the effect of varying alginate concentration on swelling behavior, gels of 1.0, 1.5, 2.0, and $2.5 \% \mathrm{LH}$ alginate with $1.5 \times$ and $2 \times \mathrm{CaCO}_{3}$ were immersed in medium with a range of $\mathrm{Ca}^{2+}$ concentrations for 2 weeks. Again, swelling occurred at low $\mathrm{Ca}^{2+}$ concentrations and shrinking occurred at high $\mathrm{Ca}^{2+}$ concentrations in the medium for all the alginate concentrations investigated [Fig. 2(b)]. At a crosslinking density of $1.5 \times \mathrm{CaCO}_{3}$, the optimal $\mathrm{Ca}^{2+}$ concentration increased with polymer concentration (Table II). In contrast, at a crosslinking density of $2 \times \mathrm{CaCO}_{3}$, gels of $1.5,2.0$, and $2.5 \% \mathrm{LH}$ alginate all maintained original wet weights at about the same $\mathrm{Ca}^{2+}$ concentration (Table II), indicating that the dimensional stability did not vary significantly with the alginate concentration at this crosslinking density.

\section{Swelling as a function of alginate chemical composition}

Our previous work demonstrated that material properties of alginate gels not only are a function of crosslinking density and polymer concentration, but also exhibit a dependency on alginate chemical composition. ${ }^{7}$ To determine whether alginate compositional variables should be considered for controlling swelling behavior as well, the effects of G-content on swelling behavior were investigated using gels made with MP and LH alginates. LH alginate has greater G-content (and longer average length of G-blocks) than MP alginate. ${ }^{7,18}$ In this study, MP alginate had higher molecular weight $\left(4.63 \times 10^{5}\right)$ than LH alginate $\left(3.73 \times 10^{5}\right)$. Gels of $1.5 \% \mathrm{MP}$ and $\mathrm{LH}$ alginates with 2 and $3 \times \mathrm{CaCO}_{3}$ were immersed in medium of $0.5-4.0 \mathrm{mM} \mathrm{Ca}{ }^{2+}$. Swelling profiles of the MP alginate gels were similar to those of the LH alginate gels [Fig. 2(c)], but

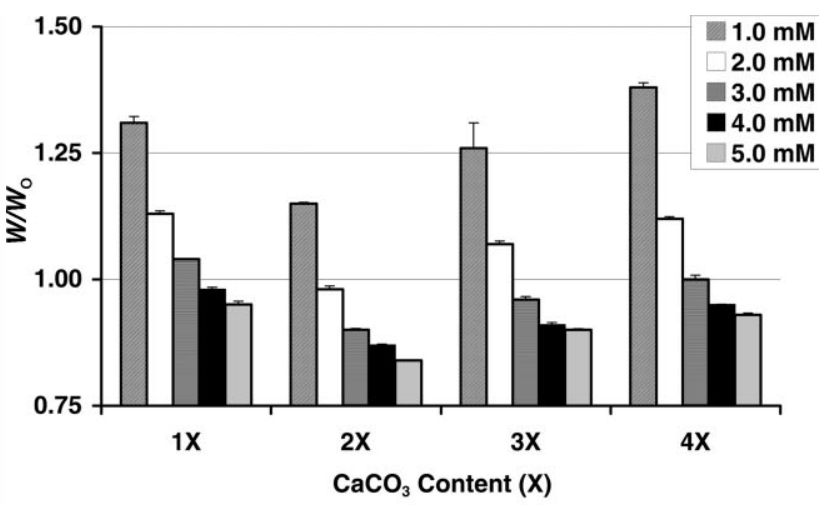

(a)

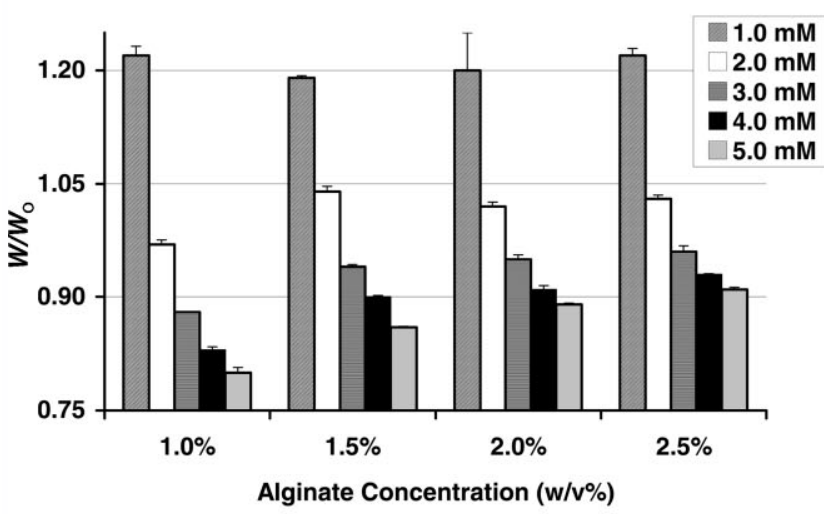

(b)

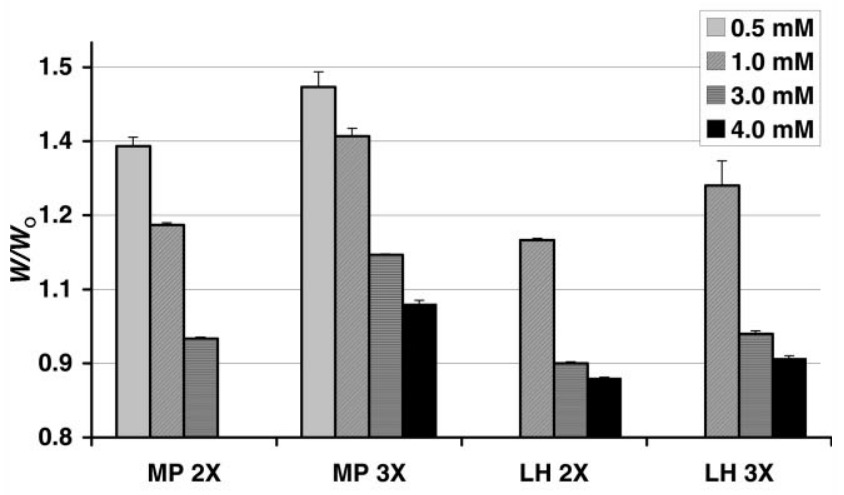

(c)

Figure 2. Swelling ratios of gels immersed in medium of varying $\mathrm{Ca}^{2+}$ concentrations for 2 weeks. The gels swelled $\left(W / W_{0}>1\right)$ at low $\mathrm{Ca}^{2+}$ concentrations and shrank (W/ $W_{\mathrm{o}}<1$ ) at high $\mathrm{Ca}^{2+}$ concentrations. (a) $1.5 \% \mathrm{LH}$ alginate gels with 2,3 , and $4 \times$ crosslinking density $\left(\mathrm{CaCO}_{3}\right.$ content of original gels); (b) $1.5 \times \mathrm{CaCO}_{3}$ gels made with 1.0, 1.5, 2.0, and $2.5 \% \mathrm{LH}$ alginate; (c) $1.5 \% \mathrm{LH}$ and $\mathrm{MP}$ alginate gels with 2 and $3 \times$ crosslinking density $\left(\mathrm{CaCO}_{3}\right.$ content of original gels). Swelling profiles of the MP alginate gels were similar to those of the LH alginate gels, with a shift upwards.

the MP alginate gels required higher $\mathrm{Ca}^{2+}$ concentrations in the medium than the LH alginate gels to maintain original dimensions (Table III), indicating 
TABLE I

Optimal $\mathrm{Ca}^{2+}$ Concentrations of Medium for $1.5 \%$ LH Alginate Gels

\begin{tabular}{cc}
\hline $\mathrm{CaCO}_{3}$ Content $(\times)$ & Optimal $\mathrm{Ca}^{2+}$ in Medium $(\mathrm{mM})$ \\
\hline 1 & 3.7 \\
1.5 & 2.4 \\
2 & 1.9 \\
3 & 2.7 \\
4 & 3.3 \\
\hline
\end{tabular}

Optimal $\mathrm{Ca}^{2+}$ concentrations of medium at which original size and shape of $1.5 \% \mathrm{LH}$ alginate gels with varying crosslinking densities $\left(\mathrm{CaCO}_{3}\right.$ content of original gels) are maintained for 2 weeks. The optimal $\mathrm{Ca}^{2+}$ concentrations were calculated from regression curves as the $\mathrm{Ca}^{2+}$ concentrations where $W / W_{\mathrm{o}}=1$.

that dimensional stability was a function of G-content (average G-block length).

Gel compositional change as a function of $\mathrm{Ca}^{+}$concentration in the medium

To understand the relationships between swelling conditions and alginate gel compositional changes, calcium and polymer contents in the gel were determined through a calcium-specific colorimetric assay and dry weight measurements, respectively. Gels of $1.5 \% \mathrm{LH}$ alginate with 2,3 , and $4 \times \mathrm{CaCO}_{3}$ were immersed in medium supplemented with either optimal $\mathrm{Ca}^{2+}$ concentrations to maintain original dimensions (Table I), or a high $\mathrm{Ca}^{2+}$ concentration of $5.0 \mathrm{mM} \mathrm{Ca}{ }^{2+}$. After 2 weeks in optimal $\mathrm{Ca}^{2+}$ medium, calcium contents of immersed gels were significantly lower than those of untreated (nonimmersed) gels ( $p \leq 0.001$; Fig. 3). Similarly, gels immersed in high $\mathrm{Ca}^{2+}$ medium for 2 weeks were found to be lower in calcium content than untreated

TABLE II

Optimal $\mathrm{Ca}^{2+}$ Concentrations of Medium for $1.5 \times$ and $2 \times \mathrm{CaCO}_{3}$ Gels

\begin{tabular}{ccc}
\hline $\begin{array}{c}\text { Alginate } \\
\begin{array}{c}\text { Concentration } \\
(\mathrm{w} / \mathrm{v} \%)\end{array}\end{array}$ & $\begin{array}{c}\text { Optimal } \mathrm{Ca}^{2+} \\
\text { in Medium } \\
(\mathrm{mM}) \text { for } \\
1.5 \times \mathrm{CaCO}_{3}\end{array}$ & $\begin{array}{c}\text { Optimal } \mathrm{Ca}^{2+} \\
\text { in Medium } \\
(\mathrm{mM}) \text { for } \\
2 \times \mathrm{CaCO}_{3}\end{array}$ \\
\hline 1.0 & 2.0 & \\
1.5 & 2.4 & 1.9 \\
2.0 & 2.4 & 1.8 \\
2.5 & 2.7 & 2.0 \\
\hline
\end{tabular}

Optimal $\mathrm{Ca}^{2+}$ concentrations of medium at which original size and shape of $1.5 \times$ and $2 \times \mathrm{CaCO}_{3}$ gels with varying alginate concentrations are maintained for 2 weeks. The optimal $\mathrm{Ca}^{2+}$ concentrations were similar across polymer concentrations for $2 \times \mathrm{CaCO}_{3}$ gels. The optimal $\mathrm{Ca}^{2+}$ concentrations were calculated from regression curves as the $\mathrm{Ca}^{2+}$ concentrations where $\mathrm{W} / W_{\mathrm{o}}=1$.
TABLE III

Optimal $\mathrm{Ca}^{2+}$ Concentrations of Medium for $1.5 \% \mathrm{LH}$ and MP Alginate Gels with 2 and $3 \times \mathrm{CaCO}_{3}$

\begin{tabular}{ccc}
\hline $\begin{array}{c}\text { Optimal } \mathrm{Ca}^{2+} \\
\mathrm{CaCO}_{3}\end{array}$ & $\begin{array}{c}\text { Optimal } \mathrm{Ca}^{2+} \\
\text { in Medium }(\mathrm{mM}) \\
\text { for MP Alginate }\end{array}$ & $\begin{array}{c}\text { in Medium }(\mathrm{mM}) \\
\text { for LH Alginate }\end{array}$ \\
\hline 2 & 2.3 & 1.9 \\
3 & 4.3 & 2.7
\end{tabular}

Optimal $\mathrm{Ca}^{2+}$ concentrations of medium at which original size and shape of $1.5 \% \mathrm{LH}$ and MP alginate gels with 2 and $3 \times \mathrm{CaCO}_{3}$ are maintained for 2 weeks. MP alginate gels required higher $\mathrm{Ca}^{2+}$ concentrations in the medium than LH alginate gels to maintain original dimensions. The optimal $\mathrm{Ca}^{2+}$ concentrations were calculated from regression curves as the $\mathrm{Ca}^{2+}$ concentrations where $\mathrm{W} / W_{\mathrm{o}}=1$.

gels $(p<0.001)$ for 3 and $4 \times \mathrm{CaCO}_{3}$ gels (Fig. 3), but no significant change in calcium content for the $2 \times$ gels. Furthermore, after immersion in high $\mathrm{Ca}^{2+}$ medium, calcium contents of 2,3, and $4 \times \mathrm{CaCO}_{3}$ gels became more similar to each other (Fig. 3). Interestingly, these results demonstrated that while optimal $\mathrm{Ca}^{2+}$ concentrations maintained original dimensions of the gels, they did not necessarily maintain composition of the gels.

Polymer content of the gels was estimated by dry $\left(W_{\mathrm{d}}\right)$ to original wet $\left(W_{\mathrm{o}}\right)$ weight ratios. $W_{\mathrm{d}} / W_{\mathrm{o}}$ of gels in both optimal and high $\mathrm{Ca}^{2+}$ medium decreased relative to untreated gels $(p<0.001)$. A decrease in $W_{\mathrm{d}} / W_{\mathrm{o}}$ indicated that loss of polymer molecules occurred when immersed in either optimal or high $\mathrm{Ca}^{2+}$ medium over the 2 weeks. In general, a greater polymer loss occurred in gels in optimal $\mathrm{Ca}^{2+}$ medium than in those immersed in

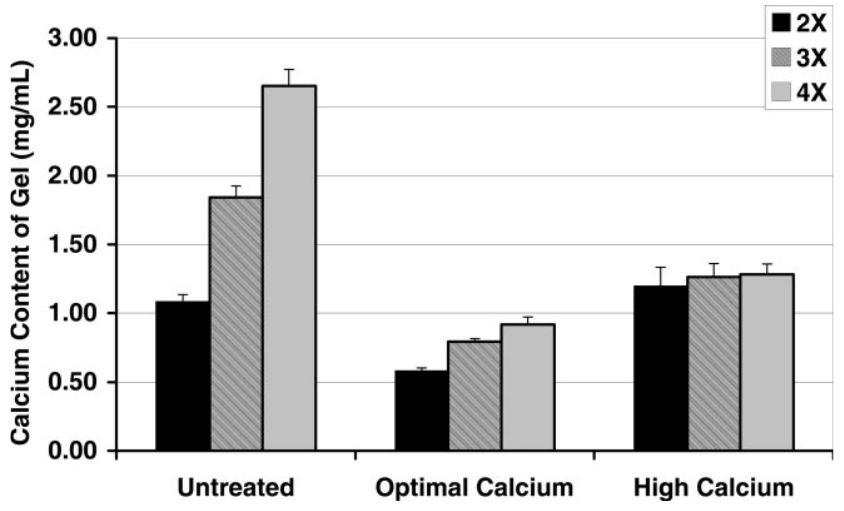

Figure 3. Calcium content of $1.5 \% \mathrm{LH}$ alginate gels with varying crosslinking densities $\left(\mathrm{CaCO}_{3}\right.$ content of original gels) before (untreated) and after 2-week immersion in medium of either optimal (to maintain original dimensions) or high $(5.0 \mathrm{mM}) \mathrm{Ca}^{2+}$ concentrations. Lower calcium contents in the gels after immersion indicated decreased crosslinking densities. The data indicates that gels immersed in medium of higher $\mathrm{Ca}^{2+}$ concentrations maintained higher crosslinking densities. 


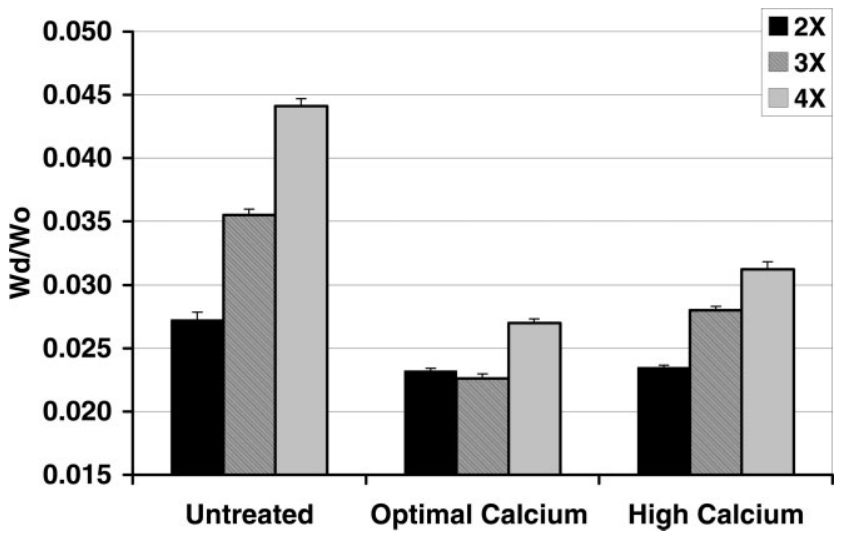

Figure 4. Dry to original-wet-weight ratios $\left(W_{\mathrm{d}} / W_{\mathrm{o}}\right)$ of $1.5 \% \mathrm{LH}$ alginate gels with varying crosslinking densities $\left(\mathrm{CaCO}_{3}\right.$ content of original gels) before (untreated) and after 2-week immersion in medium of either optimal (to maintain original gel sizes) or high $(5.0 \mathrm{mM}) \mathrm{Ca}^{2+}$ concentrations. Lower weight ratios after immersion indicated decreased polymer content. The data indicates that gels immersed in medium of high $\mathrm{Ca}^{2+}$ concentrations maintained higher polymer content.

high $\mathrm{Ca}^{2+}$ medium $\left(p<0.001\right.$ for $3 \times$ and $4 \times \mathrm{CaCO}_{3}$ gels) (Fig. 4). It appeared from these results that both a higher $\mathrm{Ca}^{2+}$ concentration in the medium and a higher initial crosslinking density of the gels had greater capacity to retain polymer molecules in the gels.

\section{Mechanical properties as a function}

\section{of calcium concentration in the medium}

To investigate how the change in gel composition during immersion affected mechanical properties, the compressive modulus and ultimate strength of the gels were measured after 2 weeks in medium. Compressive testing was performed on $1.5 \% \mathrm{LH}$ alginate gels with 2,3 , and $4 \times \mathrm{CaCO}_{3}$ after immersion in optimal $\mathrm{Ca}^{2+}$ medium to maintain original dimensions (selected from Table I). Compressive modulus and ultimate strength increased substantially with the initial crosslinking density $\left(\mathrm{CaCO}_{3}\right.$ content) of gels before immersion (untreated gels) [Fig. 5(a,b)]. After immersion in optimal $\mathrm{Ca}^{2+}$ medium, modulus and strength were significantly reduced compared with untreated gels $(p<0.002)$.

We hypothesized that higher calcium contents in the medium might counterbalance the loss in mechanical properties by retaining more polymer molecules and a higher crosslinking density. To examine the effects of a higher $\mathrm{Ca}^{2+}$ medium, $1.5 \%$ $\mathrm{LH}$ alginate gels with 2,3 , and $4 \times \mathrm{CaCO}_{3}$ were mechanically tested after 2 weeks of immersion in the medium with $5.0 \mathrm{mM} \mathrm{Ca}^{2+}$ (Fig. 5). Compressive modulus and strength of these gels were signifi- cantly higher than those in optimal $\mathrm{Ca}^{2+}$ medium $(p<0.001)$. The higher modulus and ultimate strength of gels in high $\mathrm{Ca}^{2+}$ medium, compared with those in optimal $\mathrm{Ca}^{2+}$ medium, was in agreement with the higher calcium and polymer contents of the gels. However, the improvements were different for different mechanical properties. For example, in high $\mathrm{Ca}^{2+}$ medium, the modulus of $2 \times \mathrm{CaCO}_{3}$ gels increased significantly relative to untreated gels $(p<0.001)$, while the strength remained similar to that of untreated gels $(p>0.5)$, and the modulus of $3 \times \mathrm{CaCO}_{3}$ gels did not change significantly $(p>0.2)$, while the strength decreased $(p<0.001)$ relative to untreated gels, perhaps as a result of rearrangement of the molecular network during immersion. Consistent with our hypothesis, the mechanical properties of all samples immersed in the high calcium medium $\left(5 \mathrm{mM} \mathrm{Ca}^{2+}\right)$ were significantly higher than those immersed in the "optimal" medium.

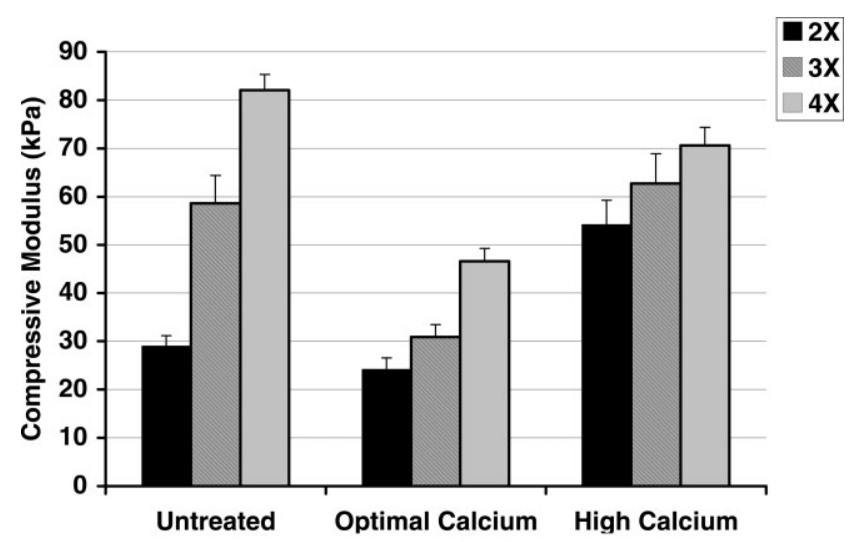

(a)

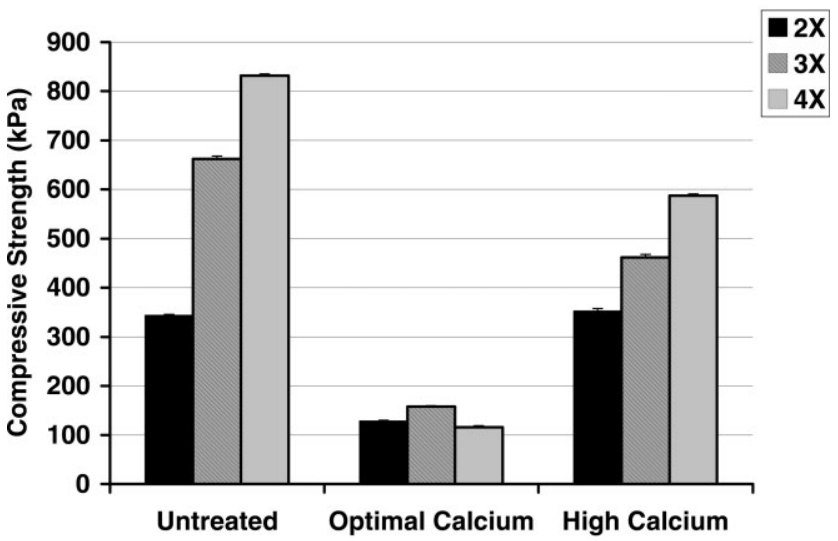

(b)

Figure 5. Mechanical properties of 1.5\% LH alginate gels with varying $\mathrm{CaCO}_{3}$ content before (untreated) and after 2-week immersion in medium of either optimal $\mathrm{Ca}^{2+}$ concentrations (to maintain original dimensions) or high $\mathrm{Ca}^{2+}$ concentrations (5.0 $\mathrm{mM} \mathrm{Ca}{ }^{2+}$ ). (a) Compressive modulus; (b) Compressive strength. 


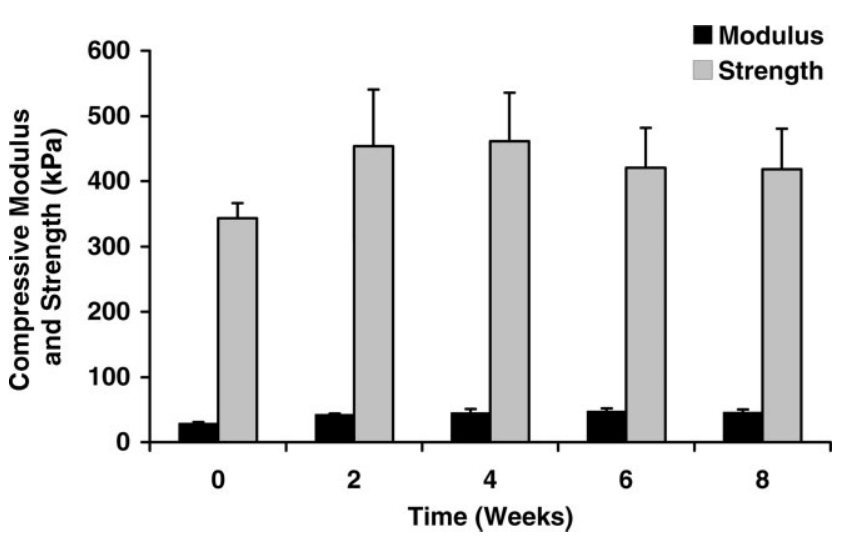

Figure 6. Compressive modulus and strength of $1.5 \% \mathrm{LH}$ alginate gels with $2 \times \mathrm{CaCO}_{3}$ during 8-week immersion in medium of $5.0 \mathrm{mM} \mathrm{Ca}{ }^{2+}$. Modulus increased between 0 and 2 weeks but remained stable thereafter with no statistical differences from 2 to 8 weeks of immersion.

Long-term mechanical properties of gels in calcium-adjusted medium

Because many biomedical applications (such as tissue engineering) require long term usage of the gels, we characterized the effects of longer term immersion on mechanical properties. Compressive modulus and strength of $1.5 \% \mathrm{LH}$ alginate gels with $2 \times \mathrm{CaCO}_{3}$ were characterized after immersion in medium with $5.0 \mathrm{mM} \mathrm{Ca}{ }^{2+}$ for up to 8 weeks (Fig. 6). As observed in the 2-week swelling experiments (Fig. 1), these gels experienced the same degrees of volume change within the first week, after which both the size and shape remained stable for the remainder of the experimental time period. Compressive modulus and strength both increased after immersion for 2 weeks and then remained relatively constant (Fig. 6). No significant difference between values was observed after 2 weeks $(p>$ 0.05). Taken together, these results demonstrated the applicability of adjusting the ionic concentration of the medium to impart long-term dimensional stability and mechanical properties to ionically crosslinked hydrogels under in vitro culture conditions.

\section{DISCUSSION}

Alginate is a biocompatible hydrogel material, and has been widely investigated as a good candidate for tissue engineering applications. ${ }^{7,16,26-29}$ However, the difficulty in generating homogeneous alginate gel-cell constructs and to maintain their dimensional and mechanical integrity poses serious challenges for tissue engineers. In our previous work, we developed techniques to generate structurally uniform alginate-cell constructs via controlling gelation rate. ${ }^{7}$ In this work, we developed a method to control the dimensional stability and the mechanical properties of ionically crosslinked hydrogels in a tissue culture environment (an aqueous medium).

Several effects are involved in the swelling behavior of ionically crosslinked hydrogels. The free energy of mixing of the polymer chains with the medium will drive the gel to swell because thermodynamically the alginate molecules tend assume an open structure to increase interactions of carboxyl and hydroxyl groups with water molecules. ${ }^{30-32}$ Further, the osmotic pressure created by the presence of mobile counterions in the hydroge ${ }^{30-32}$ will cause water molecules from the medium to diffuse into the gel to balance chemical potentials, leading to swelling. There is also the added complexity of crosslinking ions being displaced by noncrosslinking ions, potentially leading to complete dissociation of the gel. In contrast, crosslinking creates elastic forces in the gel network ${ }^{31,33}$ that provide resistance to swelling tendencies.

In our studies, "optimal" $\mathrm{Ca}^{2+}$ concentration in the medium increased with initial crosslinking density $\left(\mathrm{CaCO}_{3}\right.$ content of the original gels $)$ and alginate concentration. Increasing the original crosslinking density of a gel resulted in greater elastic forces to resist swelling. However, higher crosslinking density also led to a greater concentration of counterions within the gel, resulting in increased tendency of water molecules to diffuse into the gel. Supplementing the solution with calcium ions may have balanced the relative concentrations of crosslinking to noncrosslinking ions to control swelling. With regard to alginate concentration, higher polymer concentration may have led to greater swelling tendency because of the hydrophilicity of alginate molecules. However, higher alginate concentration also resulted in the involvement of more alginate molecules in crosslinking and entanglements, leading to higher elastic forces opposing swelling. Interestingly, at a crosslinking density of $2 \times \mathrm{CaCO}_{3}$, the enhanced elastic forces resulting from increased polymer concentration seemed to balance the swelling tendency (Table II). This may allow us to vary polymer concentration to advantageously tailor diffusion and mechanical properties without varying the calcium content of the culture medium.

Our results clearly indicate that swelling behavior is a function of initial crosslink density, alginate concentration, and alginate chemical composition. Optimal $\mathrm{Ca}^{2+}$ concentrations to maintain original dimensions of high $G$ content alginate gels were lower than those of high $\mathrm{M}$ content, despite higher molecular weight of the latter samples $\left(3.73 \times 10^{5}\right.$ vs. $4.63 \times 10^{5}$, respectively). While it was expected that longer and more entangled polymer molecules 
would provide greater resistance to swelling, these results indicate that the structure of the alginate molecule affected the swelling behavior of the gel more significantly. Previously, we demonstrated that G-blocks allow more effective crosslink formation to increase mechanical properties of the gels. ${ }^{7}$ Taken together, these results are consistent with other reports that crosslinked G-blocks associate laterally to various multiplicities, forming more highly ordered regions, ${ }^{34}$ and that structural integrity and mechanical properties are highly dependent on crosslinking of G-blocks. ${ }^{35}$ Smidsrod and Skj demonstrated more effective crosslinking to occur with the carboxyl groups of G-blocks than M- or MG-blocks. ${ }^{35}$ We speculate in our current studies that calcium ions involved in crosslink junctions formed with G-blocks were less susceptible to decrosslinking because of the "egg-box" conformation and associated highly ordered regions. Indeed, in separate studies, both Wang and Thu suggested that in an aqueous environment, calcium ion decrosslinking occurs first with M- and MG-blocks in alginate gels. ${ }^{31,33}$ In our studies, the less effective crosslinking of the higher M-content MP alginate gels may have resulted in weaker elastic forces, and thus greater swelling tendencies. A higher $\mathrm{Ca}^{2+}$ concentration in the solution was necessary for MP alginate gels to counterbalance the swelling tendencies. Taken together, these results demonstrate a greater effect of G-content (and longer average length of the G-blocks) than of molecular entanglement resulted from a higher molecular weight.

Dimensional stability achieved by controlling the $\mathrm{Ca}^{2+}$ concentration of the medium was accompanied by decreases in calcium and polymer contents during immersion, suggesting reduced crosslinking density and polymer concentration. Though gels in optimal $\mathrm{Ca}^{2+}$ medium maintained their original dimensions, the mechanical properties were decreased in general. We previously demonstrated mechanical properties of homogeneous alginate gels to be a function of both crosslinking density and polymer concentration. ${ }^{7}$ Thus, the decreases in both calcium content and $W_{\mathrm{d}} / W_{\mathrm{o}}$ ratio suggest that the decreased mechanical properties resulted from lower crosslink density and polymer concentration. However, for tissue engineering applications, cells synthesize their own extracellular matrix, which may balance the loss of mechanical properties or improve the mechanical properties over time. This phenomenon has been previously demonstrated in other hydrogel systems. ${ }^{36,37}$

In an 8-week study, we showed that dimensional stability was reached within the first week. In addition, the high $\mathrm{Ca}^{2+}$ medium either maintained or improved mechanical properties of the gels as measured after 2 weeks of immersion. Furthermore, their mechanical properties did not change substantially from 2 to 8 weeks, indicating that the gels reached a stable equilibrium with the controlled aqueous environment within the first 2 weeks.

In summary, we have demonstrated a novel method to successfully control the dimensions and mechanical properties of ionically crosslinked alginate hydrogels by controlling the calcium ion concentration in the aqueous environment. Our data suggests that crosslinking density and polymer content both change within the gel to achieve equilibrium with the ionic concentration of the external environment. These studies contribute to the current understanding of the mechanisms of swelling of ionically crosslinked hydrogels in a physiological environment, and do so by probing the equilibrium of the system subjected to specific ionic concentrations of the external environment. This system significantly enhances the potential of ionically crosslinked alginate hydrogels for a variety of in vitro biomedical applications, such as tissue engineering scaffolding and drug or biofactor delivery.

The authors wish to acknowledge the following people for their assistance: Sargum Manley, Natasha Gosek, Ami Shah, Khan Owais Akmal and Kathryn Swan.

\section{References}

1. Hoffman AS. Hydrogels for biomedical applications. Adv Drug Deliv Rev 2002;54:3-12.

2. Ma PX. Scaffolds for tissue fabrication. Mater Today 2004; 7:30-40.

3. Ma PX. Tissue engineering. In: Kroschwitz JI, editor. Encyclopedia of Polymer Science and Technology. Hoboken, NJ: Wiley; 2005. p 261-291. www.mrw.interscience.wiley.com/ epst.

4. Nguyen KT, West JL. Photopolymerizable hydrogels for tissue engineering applications. Biomaterials 2002;23:4307-4314.

5. Elisseeff J. Injectable cartilage tissue engineering. Expert Opin Biol Ther 2004;4:1849-1859.

6. Peppas NA, Bures P, Leobandung W, Ichikawa H. Hydrogels in pharmaceutical formulations. Eur J Pharm Biopharm 2000; 50:27-46.

7. Kuo CK, Ma PX. lonically crosslinked alginate hydrogels as scaffolds for tissue engineering. I. Structure, gelation rate and mechanical properties. Biomaterials 2001;22:511-521.

8. Nuttelman CR, Henry SM, Anseth KS. Synthesis and characterization of photocrosslinkable, degradable poly(vinyl alcohol)-based tissue engineering scaffolds. Biomaterials 2002;23: 3617-3626.

9. Li Q, Wang J, Shahani S, Sun DD, Sharma B, Elisseeff JH, Leong KW. Biodegradable and photocrosslinkable polyphosphoester hydrogel. Biomaterials 2006;27:1027-1034.

10. Li Q, Williams CG, Sun DD, Wang J, Leong K, Elisseeff JH. Photocrosslinkable polysaccharides based on chondroitin sulfate. J Biomed Mater Res A 2004;68:28-33.

11. Burdick JA, Chung C, Jia X, Randolph MA, Langer R. Controlled degradation and mechanical behavior of photopolymerized hyaluronic acid networks. Biomacromolecules 2005; 6:386-391. 
12. Temenoff JS, Athanasiou KA, LeBaron RG, Mikos AG. Effect of poly(ethylene glycol) molecular weight on tensile and swelling properties of oligo(poly(ethylene glycol) fumarate) hydrogels for cartilage tissue engineering. J Biomed Mater Res 2002;59:429-437.

13. Bryant SJ, Anseth KS, Lee DA, Bader DL. Crosslinking density influences the morphology of chondrocytes photoencapsulated in PEG hydrogels during the application of compressive strain. J Orthop Res 2004;22:1143-1149.

14. Bryant SJ, Anseth KS. Hydrogel properties influence ECM production by chondrocytes photoencapsulated in poly(ethylene glycol) hydrogels. J Biomed Mater Res 2002;59:63-72.

15. Bryant SJ, Durand KL, Anseth KS. Manipulations in hydrogel chemistry control photoencapsulated chondrocyte behavior and their extracellular matrix production. J Biomed Mater Res A 2003;67:1430-1436.

16. Ma PX. Alginate for tissue engineering. In: Ma PX, Elisseeff J, editors. Scaffolding in Tissue Engineering. Boca Raton, FL: CRC Press; 2005. p 13-25.

17. Draget KI, Ostgaard K, Smidsrod O. Homogeneous alginate gels: A technical approach. Carbohydr Polym 1991;14:159178.

18. Martinsen A, Skjåk-Bræk G, Smidsrød O. Alginate as immobilization material. I. Correlation between chemical and physical properties of alginate gel beads. Biotechnol Bioeng 1989; 33:79-89.

19. Grant GT, Morris ER, Rees DA, Smith PJC, Thom D. Biological interactions between polysaccharides and divalent cations-Egg-Box model. Febs Lett 1973;32:195-198.

20. Rees DA. Polysaccharide shapes and their interactions-Some recent advances. Pure Appl Chem 1981;53:1-14.

21. Skjakbraek G, Smidsrod O, Larsen B. Tailoring of alginates by enzymatic modification invitro. Int J Biol Macromol 1986; 8:330-336.

22. Aebischer P, Buchser E, Joseph J, Favre J, de TN, Lysaght M, Rudnick S, Goddard M. Transplantation in humans of encapsulated xenogeneic cells without immunosuppression: A preliminary report. Transplantation 1994;58:1275-1277.

23. Atala A, Kim W, Paige K, Vacanti C, Retik A. Endoscopic treatment of vesicoureteral reflux with a chondrocyte-alginate suspension. J Urol 1994;152(2, Part 2):641-643; discussion 644.

24. Skjåk-Bræk G, Grasdalen H, Smidsrød O. Inhomogeneous polysaccharide ionic gels. Carbohydr Polym 1989;10:31-54.
25. LeRoux MA, Guilak F, Setton LA. Compressive and shear properties of alginate gel: Effects of sodium ions and alginate concentration. J Biomed Mater Res 1999;47:46-53.

26. Williams GM, Klein TJ, Sah RL. Cell density alters matrix accumulation in two distinct fractions and the mechanical integrity of alginate-chondrocyte constructs. Acta Biomater 2005;1:625-633.

27. Elkayam T, Amitay-Shaprut S, Dvir-Ginzberg M, Harel T, Cohen S. Enhancing the drug metabolism activities of $\mathrm{C} 3 \mathrm{~A}-$ A human hepatocyte cell line-by tissue engineering within alginate scaffolds. Tissue Eng 2006;12:1357-1368.

28. Hill E, Boontheekul T, Mooney DJ. Designing scaffolds to enhance transplanted myoblast survival and migration. Tissue Eng 2006;12:1295-1304.

29. Cohen DL, Malone E, Lipson H, Bonassar LJ. Direct freeform fabrication of seeded hydrogels in arbitrary geometries. Tissue Eng 2006;12:1325-1335.

30. Moe ST, Skjakbraek G, Elgsaeter A, Smidsrod O. Swelling of covalently cross-linked alginate gels-Influence of ionic solutes and nonpolar-solvents. Macromolecules 1993;26:3589-3597.

31. Wang XW, Spencer HG. Calcium alginate gels: Formation and stability in the presence of an inert electrolyte. Polymer 1998;39:2759-2764.

32. Saitoh S, Araki Y, Kon R, Katsura H, Taira M. Swelling/ deswelling mechanism of calcium alginate gel in aqueous solutions. Dent Mater J 2000;19:396-404.

33. Thu B, Bruheim P, Espevik T, Smidsrod O, SoonShiong P, SkjakBraek G. Alginate polycation microcapsules. I. Interaction between alginate and polycation. Biomaterials 1996;17: 1031-1040.

34. Stokke BT, Draget KI, Smidsrod O, Yuguchi Y, Urakawa H, Kajiwara K. Small-angle X-ray scattering and rheological characterization of alginate gels. I. Ca-alginate gels. Macromolecules 2000;33:1853-1863.

35. Smidsrod O, Skj a-BG. Alginate as immobilization matrix for cells. Trends Biotechnol 1990;8:71-78.

36. Buschmann MD, Gluzband YA, Grodzinsky AJ, Kimura JH, Hunziker EB. Chondrocytes in agarose culture synthesize a mechanically functional extracellular matrix. J Orthop Res 1992;10:745-758.

37. Elisseeff J, McIntosh W, Anseth K, Riley S, Ragan P, Langer R. Photoencapsulation of chondrocytes in poly(ethylene oxide)-based semi-interpenetrating networks. J Biomed Mater Res 2000;51:164-171. 